\title{
Control of hepatocellular apoptosis by cytokines (TGF- $\beta$ I), liver tumor promoter (phenobarbital) and nutritional factors (glucose): approach to validate the hepatoma cell line HCC-I.2 as cell culture model
}

\author{
Anneliese Karwan, Julia Dornetshuber, Rolf Schulte-Hermann and \\ Wilfried Bursch*
}

Address: Department of Medicine I, Institute of Cancer Research, Research Unit Toxicology and Prevention, Medical University of Vienna, Austria

Email: Wilfried Bursch* - wilfried.bursch@meduniwien.ac.at

* Corresponding author

\author{
from 13th Scientific Symposium of the Austrian Pharmacological Society (APHAR). Joint Meeting with the Austrian Society of Toxicology (ASTOX) and the \\ Hungarian Society for Experimental and Clinical Pharmacology (MFT) \\ Vienna, Austria. 22-24 November 2007 \\ Published: I 4 November 2007 \\ BMC Pharmacology 2007, 7(Suppl 2):A63 doi:10.1 186/I47|-22 I0-7-S2-A63
}

This abstract is available from: http://www.biomedcentral.com/I47I-22 I0/7/S2/A63

(C) 2007 Karwan et al; licensee BioMed Central Ltd.

Since a long line of years we have studied liver growth regulation (cell proliferation, apoptosis) in vivo. One of the prominent findings was that TGF- $\beta 1$ constitutes a major death signal in rat liver, acting in concert with nutritional factors to maintain liver cell number homeostasis. Toxicological tests with rodents in vivo showed that liver tumor promoter (e.g. phenobarbital) favour cell multiplication and inhibit apoptosis, thereby accelerating hepatocarcinogenesis. To elucidate their mode of action (MOA), along with the general need for alternative test models in toxicology, we searched for a liver cell culture system yielding a high concordance with our in vivo findings. Here we report on a newly established cell line HCC-1.2 [Grasl-Kraupp et al., submitted] exhibiting the following features: (1) a high sensitivity towards the anti-proliferative and pro-apoptotic action of TGF- $\beta 1$; (2) apoptosis is mediated via the intrinsic pathway as demonstrated by caspase analysis; (3) inhibition of TGF- $\beta 1$-induced apoptosis by liver tumor promoter, as exemplified by phenobarbital; (4) glucose withdrawal exerts an additive effect to the pro-apoptotic action of TGF- $\beta 1$, all of which agree with our previous in vivo observations on liver growth regulation. Thus, our observations suggest that the HCC1.2 cells constitute a valid test system as it meets well with prerequists for further studies tackling the MOA of liver tumor promoter, along with their interaction with nutritional factors. 